\title{
Resonance reactions and enhancement of weak interactions in collisions of cold molecules
}

\author{
V.V. Flambaum and J.S.M. Ginges \\ School of Physics, University of New South Wales, Sydney 2052, Australia
}

(Dated: November 4, 2018)

\begin{abstract}
With the creation of ultracold atoms and molecules, a new type of chemistry - "resonance" chemistry - emerges: chemical reactions can occur when the energy of colliding atoms/molecules matches a bound state of the combined molecule (Feshbach resonance). This chemistry is rather similar to reactions that take place in nuclei at low energies. In this paper we suggest some problems for future experimental and theoretical work related to the resonance chemistry of ultracold molecules. Molecular Bose-Einstein condensates (BECs) are particularly interesting because in this system collisions and chemical reactions are extremely sensitive to weak fields; also, a preferred reaction channel may be enhanced due to a finite number of final states. The sensitivity to weak fields arises due to the high density of narrow compound resonances and the macroscopic number of molecules with kinetic energy $E=0$ (in the ground state of a mean-field potential). The high sensitivity to the magnetic field may be used to measure the distribution of energy intervals, widths, and magnetic moments of compound resonances and study the onset of quantum chaos. A difference in the production rate of right-handed and left-handed chiral molecules may be produced by external electric $\mathbf{E}$ and magnetic $\mathbf{B}$ fields and the finite width $\Gamma$ of the resonance (correlation $\Gamma \mathbf{E} \cdot \mathbf{B}$ ). The same effect may be produced by the parity-violating energy difference in chiral molecules.

PACS numbers: 03.75.Nt, 82.30.Cf, 32.80.Ys, 33.55.Ad
\end{abstract}

\section{INTRODUCTION}

Techniques to produce and manipulate ultracold atoms and molecules are rapidly developing. Bose-Einstein condensation (BEC) has been realized in numerous dilute atomic gases [1] and, recently, in molecules [2]. In these systems a new type of chemistry - "resonance chemistry" - emerges. Chemical reactions can take place when the energy of the free atoms/molecules matches the energy of a bound state of the combined system due to resonant coupling between the free and bound states. The relative energies of the states can be tuned by variation of a magnetic field. Ultracold molecules have been formed from degenerate Fermi gases [3] and atomic BECs [4] at Feshbach resonances [5, [6], where two free particles resonantly couple to a bound state of the combined system. Just recently, the resonant coupling of three free atoms to a bound state of a triatomic molecule (Efimov resonance) has been observed [7]. The aim of this short paper is to attract attention to some interesting problems related to resonance chemistry in ultracold molecules. Some results have already been presented in our preprint [8].

\section{DENSE SPECTRUM OF COMPOUND STATES, ENHANCEMENT OF WEAK PERTURBATIONS, AND QUANTUM CHAOS}

The collisions of atoms in a BEC can be controlled by the variation of a relatively weak magnetic field; the magnitude and sign of the scattering length can be changed by varying the field near a Feshbach resonance [5, 9]. In this way the expansion and collapse of an atomic BEC has been observed [10]. In molecules, a change in the scattering length can be achieved with a magnetic field much weaker than that used to obtain the same effect in atoms. This increase in the sensitivity to weak fields is due to the much richer spectra of molecules: there is an exponential increase of the density of resonances with the number of "active" particles.

Consider, for example, the formation of an intermediate non-stationary compound state of four atoms arising from the collision of two cold diatomic molecules. This state may be considered as an excited state of the four-atom molecule (if this molecule has a finite binding energy). Compound states in molecules combine many electronic, vibrational, rotational, and hyperfine (spins of nuclei) degrees of freedom. Naturally, the interval between these compound levels is very small. Even without nuclear spins, the density of states may exceed $10^{5}$ states per $1 \mathrm{~K}$ energy interval $\left(2 \times 10^{5} \mathrm{~K}^{-1}\right.$ for collision of two $\mathrm{PbO}$ molecules 11]). Simultaneously, the decay width of these complex compound states decreases since the emitted particle (atom or electron) must collect the energy from many degrees of freedom to have enough energy to escape from a potential well produced by the interaction with other particles forming the compound state. Similarly, in radiative decay one electron must collect energy from all degrees of freedom to radiate an energetic photon. This phenomenon of exponential decrease of the intervals between energy levels and decrease of the decay widths is well-known in nuclear physics.

Determination of the energy intervals, widths, and magnetic moments of resonances in cold molecular collisions is an interesting problem by itself and may help to find out if there is "quantum chaos" in this problem (a discussion of chaos in molecular collisions may be found, for example, in Ref. 11] and references therein). Conven- 
tional signatures of chaos include Wigner-Dyson statistics of energy intervals between compound states, PorterThomas statistics of capture and decay widths, statistics of magnetic moments, etc. (see, e.g., the review [12]).

Indeed, the spectra and wave functions of compound states in nuclei are usually chaotic. This is because the residual interaction between the particles exceeds the interval between the energy levels. Therefore, the wave function of a compound state becomes a superposition of a huge number of basic components (Slater determinants built from products of single-particle orbitals). The expansion coefficients in these linear combinations of basic components behave like "random variables". Quantum chaos also appears in excited states of some rare-earth and actinide atoms and in many multiply-charged ions 13]. In cold molecular collisions, compound states should also be chaotic superpositions of a large number of basic components (a basic component here is a state which may be classified using certain hyperfine, rotational, vibrational, and electronic quantum numbers).

In nuclei the statistics pertaining to quantum chaos are obtained by changing the energy of the initial particles. In cold molecular collisions the statistics may be studied by applying a magnetic field which changes the positions of the compound resonances at a given energy. A very interesting manifestation of quantum chaos is the enhancement of weak interaction effects (see, e.g., reviews 14] and references within).

\section{DIFFERENCE IN PRODUCTION RATES OF CHIRAL MOLECULES}

\section{A. Parity violating weak interaction}

The high sensitivity of ultracold molecules to weak fields could be used to search for an energy difference between chiral molecules produced by the parity violating (PV) weak interaction. As we will see, this difference leads to a difference in the production rate of right-handed and left-handed chiral molecules. The interest in these problems with chiral molecules is mainly motivated by attempts to find a mechanism which produced homo-chiral biological molecules and to obtain a better understanding of biological evolution. It is wellknown that biological molecules have a definite chiral structure (for example, there are only naturally occurring left-handed amino acids and right-handed sugars) [15]. There have been numerous attempts to explain this effect by the influence of the PV weak interaction, which breaks the energy equivalence of right- and left-handed molecules 16, 17]. That parity violation can discriminate between molecules of different chirality is easily seen: the PV electron-nucleus interaction in atoms [18, 19] creates a spin helix of the electrons which interacts differently with right- and left-handed molecules. However, the parity violating energy difference $\Delta E_{P V}$ is very small [20],

$$
\Delta E_{P V} \sim 10^{-20} Z^{5} \eta \text { a.u. , }
$$

where $Z$ is the nuclear charge of the heaviest atom, and $\eta$ is an asymmetry factor which can be found from molecular structure calculations. This strong dependence on $Z$ originates from the weak $\left(\propto Z^{3}\right)$ and spin-orbit $\left(\propto Z^{2}\right)$ interactions. It may appear that in molecules with heavy atoms $\Delta E_{P V}$ could become relatively large due to the $Z^{5}$ dependence. However, the asymmetry factor $\eta$ remains very small. The effect may be orders of magnitude larger for molecules with two heavy atoms 20]. For calculations of $\Delta E_{P V}$ for various molecules, see, e.g., 21] and references therein. So far a PV energy difference in molecules has eluded detection (see, e.g., 22]).

Let us consider how a PV energy difference could manifest itself in the collision of two ultracold molecules. Remember that in order to form a chiral molecule there must be at least four atoms involved [23]; therefore, the collision of two diatomic molecules is sufficient. The cross-section for formation of a chiral compound molecular state due to $s$-wave scattering can be expressed, using the Breit-Wigner formula, as

$$
\sigma=\frac{\pi}{k^{2}} \frac{\Gamma_{c} \Gamma}{\left(E-E_{0}\right)^{2}+\Gamma^{2} / 4},
$$

where $k$ is the wave vector, $\Gamma_{c}$ is the capture width, and $\Gamma$ is the total width of the resonance. The PV weak interaction in the chiral molecules shifts the resonance energies. For example, let's consider that for the left-handed structure $E_{0} \rightarrow E_{L}=E_{0}-\Delta E_{P V} / 2$ while for the righthanded structure $E_{0} \rightarrow E_{R}=E_{0}+\Delta E_{P V} / 2$. Therefore, cross-sections for the formation of left and right molecules, $\sigma_{L}$ and $\sigma_{R}$, from achiral components may be different. We can define an asymmetry parameter

$$
P=\frac{\sigma_{R}-\sigma_{L}}{\sigma_{R}+\sigma_{L}} .
$$

The maximum value for $P$ is reached when $E=E_{0} \pm \Gamma / 2$. At this energy the asymmetry parameter

$$
\left|P_{\max }\right|=\frac{\Delta E_{P V}}{\Gamma} .
$$

In principle, the resonances can be shifted to the point of maximum $P, E_{0} \pm \Gamma / 2 \approx 0$, by application of an external electric or magnetic field.

A difference in the number of right-handed and lefthanded chiral molecules, proportional to the asymmetry parameter $P$, produces optical activity. This may be a method of detection of the PV effect. Note that PV experiments with atoms have already demonstrated a very high sensitivity to small angles of rotation of the light polarization plane [18, 19]. One may also try to detect the circular polarization of light emitted in the decay of compound states of chiral molecules. Indeed, light emitted by a chiral molecule has a certain degree of circular polarization. Therefore, a small difference in the number of right-handed and left-handed molecules produces some very small circular polarization.

Let us briefly consider what we may expect for the size of the optical rotation. The angle of rotation $\varphi$ of 
the polarization plane of light passing through the sample may be expressed in terms of the asymmetry parameter $P$ as

$$
\varphi=\frac{N_{R}}{N_{R}+N_{L}} \varphi_{R}+\frac{N_{L}}{N_{R}+N_{L}} \varphi_{L}=P \varphi_{R},
$$

where $N_{R}, N_{L}$ are the numbers of right- and left-handed molecules and $\varphi_{R}, \varphi_{L}=-\varphi_{R}$ are the angles of rotation that the respective molecules produce. We use Eq. (4) to estimate $P$. The largest values for $\Delta E_{P V}$ that have been considered in molecular calculations are $\sim 10^{4} \mathrm{~Hz}$ (e.g., for $\mathrm{H}_{2} \mathrm{Po}_{2}$ 21] ). The width of the level $\Gamma$ may be quite small, since the capture width $\Gamma_{c}=0$ for energy $E=0$ and the radiative width may be much smaller than $10^{6} \mathrm{~Hz}$ (a typical width for optical photon emission) because of "chaotic" suppression discussed at the beginning of this paper. One should check if there are additional decay channels: the four-atom compound state may have enough energy to decay by emission of one atom or electron. The actual value of the width, which also includes a width due to collisions, should be determined experimentally. In ${ }^{133} \mathrm{Cs}$, very narrow widths, as small as $3.5 \mathrm{kHz}$, have been observed in $g$-wave Feshbach resonances [24]. Therefore, an asymmetry parameter $P \sim 1$ is not out of the question. The value for the rotation angle $\varphi_{R}$ depends on a number of parameters: the molecular density, the refractive index, the pseudoscalar polarizability, the wavelength of the light, and the pathlength [25, 26]. A well-known example of a chiral molecule is sucrose; in water solution, it rotates light at the sodium D-line by an angle $\sim 700 \rho \mathrm{deg} / \mathrm{m}$, where $\rho$ is the density (or concentration) in $\mathrm{g} / \mathrm{cm}^{3}$. The numerical coefficient is an intensive property of the molecule, depending on the temperature of the sample and the wavelength of light. The value for sucrose is not untypical. We can expect similar values for chiral molecules in gases, i.e. a rotation angle $\sim 1 \mathrm{deg} / \mathrm{m}$. In a very low-density gas the angle of rotation can be significantly increased by tuning the light frequency to resonance.

In collisions in molecular BECs there may be some enhancement of the effect due to the macroscopic number of molecules in the ground state with vanishingly small energy spread. Moreover, the effect may be Bose-enhanced (non-linear enhancement of the reaction due to the number of identical particles in the final state). Through a small difference in reaction rates such enhancement can lead to an almost complete selectivity of one reaction channel over another [27]. This could be a mechanism for selectivity of chiral molecules of one handedness produced, e.g., by a parity violating energy difference or the fields $\mathbf{E} \cdot \mathbf{B}$ (see the following section).

\section{B. Pseudoscalar correlation produced by electric and magnetic fields}

Constant homogeneous fields cannot produce an energy difference between molecules of different chirality.
Indeed, a chiral molecule is characterized by a pseudoscalar $\left[\mathbf{n}_{\mathbf{A}} \times \mathbf{n}_{\mathbf{B}}\right] \cdot \mathbf{n}_{\mathbf{C}}$, where $\mathbf{n}_{\mathbf{A}}, \mathbf{n}_{\mathbf{B}}$, and $\mathbf{n}_{\mathbf{C}}$ are vectors showing locations of atoms $A, B$, and $C$ relative to atom $D$. The weak interaction is proportional to a pseudoscalar $\mathbf{s} \cdot \mathbf{p}$, where $\mathbf{s}$ and $\mathbf{p}$ are the electron spin and momentum. This interaction produces chirality-dependent energy shifts since its pseudoscalar $\mathbf{s} \cdot \mathbf{p}$ may be correlated with the molecular pseudoscalar $\left[\mathbf{n}_{\mathbf{A}} \times \mathbf{n}_{\mathbf{B}}\right] \cdot \mathbf{n}_{\mathbf{C}}$ (with the help of the spin-orbit interaction which links the spin $\mathbf{s}$ with the coordinate variables). To imitate the PV energy difference we need to make a $T$-invariant pseudoscalar (effective interaction) from electric and magnetic fields. All combinations like $\mathbf{E} \times \mathbf{B}, \mathbf{E} \cdot \mathbf{B}, \mathbf{E} \cdot \mathbf{E}$, and $\mathbf{B} \cdot \mathbf{B}$ do not satisfy this requirement. The molecular vectors $\mathbf{n}_{\mathbf{A}}, \mathbf{n}_{\mathbf{B}}$, and $\mathbf{n}_{\mathbf{C}}$ cannot be included into these effective interactions since they disappear after averaging over molecular orientations. In principle, one can make the $T$-even pseudoscalar by considering inhomogeneous and time-dependent fields $\left(\mathbf{B} \cdot \frac{\partial \mathbf{E}}{\partial t}, \mathbf{E} \cdot \frac{\partial \mathbf{B}}{\partial t}\right.$, etc.). However, the corresponding effects should be very small (proportional to the change of the field on the molecular scale). Therefore, stray fields can hardly imitate effects of the $\mathrm{PV}$ weak interaction.

The only exception here may be the correlation $\mathbf{E} \cdot \mathbf{B}$. It violates both $P$ and $T$ invariance, therefore it cannot produce an energy shift of a stationary state. However, a compound resonance is not a stationary state. Therefore, this correlation can induce a difference in the production of right-handed and left-handed chiral molecules due to the finite width of the compound state or due to any final state interaction in general. In principle, this may be a natural source of asymmetry between biological chiral molecules. A reliable way to find the magnitude of the effects is to perform a dedicated experiment with controlled fields.

\section{Origin of the T-odd correlation in circular polarization of photons}

It is important to show how the $T$-odd correlation $\mathbf{E} \cdot \mathbf{B}$ can produce $T$-even effects. We will consider a simpler effect produced by these fields: circular polarization of photons in atomic transitions. This possibility was first pointed out for hydrogen in Ref. [28].

The magnetic field alone can produce circular polarization for photons emitted in a definite direction. This effect has the same origin as Faraday rotation and can be described by the correlation $\mathbf{B} \cdot \mathbf{k} \lambda$, where $\mathbf{k}$ is the unit momentum vector and $\lambda$ is the helicity (photon states with definite circular polarization correspond to $\lambda= \pm 1$ ). Such circular polarization disappears after averaging over photon directions. The case of two fields with non-zero $\mathbf{E} \cdot \mathbf{B}$ is different. Here the circular polarization does not vanish after averaging. Indeed, the helicity (circular polarization) of a particle $\lambda=\mathbf{s} \cdot \mathbf{k}$ is a $T$-even pseudoscalar (s is the unit spin vector). As known in atomic transitions, a small photon circular polarization is nor- 
mally produced by the weak interaction which is also a $T$-even pseudoscalar. The correlation $\mathbf{E} \cdot \mathbf{B}$ is a $T$-odd pseudoscalar, therefore it may create circular polarization proportional to the widths of the involved quasistationary states or any final state interactions. Thus, the situation with the production of helicity/circular polarization is similar to the situation with the production of chirality.

The result for the circular polarization $P$ can be schematically presented using the following notations. Let us assume that an atomic electron is excited from a ground state to a non-stationary state $p_{1 / 2}$ with energy $E_{1 / 2}$, width $\Gamma_{1 / 2}$, and excitation amplitude $T_{1 / 2}$. Then this state decays to some final state $f$ and emits a photon with real radiation amplitude M1. The amplitude of this process can be presented as

$$
A=\frac{T_{1 / 2} M 1}{E-E_{1 / 2}+i \Gamma_{1 / 2} / 2} .
$$

Here $E$ is the excitation energy. We assume that $E \approx$ $E_{1 / 2}$. Assume that close to the $p_{1 / 2}$ state there are states $p_{3 / 2}$ and $s_{1 / 2}$. The state $s_{1 / 2}$ may decay to the same final state with imaginary radiation amplitude $i E 1$ (origin of the imaginary unit $i$ in this formula may be found, e.g., in the book [18]).

The combined effect of the magnetic and electric field gives us another amplitude:

$$
B=\frac{T_{1 / 2}\left\langle p_{1 / 2}|\boldsymbol{\mu} \cdot \mathbf{B}| p_{3 / 2}\right\rangle\left\langle p_{3 / 2}|e \mathbf{r} \cdot \mathbf{E}| s_{1 / 2}\right\rangle i E 1}{\left(E-E_{\frac{1}{2}}+\frac{i \Gamma_{\frac{1}{2}}}{2}\right)\left(E-E_{\frac{3}{2}}+\frac{i \Gamma_{\frac{3}{2}}}{2}\right)\left(E-E_{s}+\frac{i \Gamma_{s}}{2}\right)} .
$$

We may assume here that both fields $\mathbf{B}$ and $\mathbf{E}$ are directed along the $z$-axis. Then summation over the angular momenta of the intermediate states gives a trivial positive coefficient.

For comparison we present here the amplitude $B^{W}$ produced by the weak interaction:

$$
B^{W}=\frac{T_{1 / 2}\left\langle p_{1 / 2}|W| s_{1 / 2}\right\rangle i E 1}{\left(E-E_{1 / 2}+i \Gamma_{1 / 2} / 2\right)\left(E-E_{s}+i \Gamma_{s} / 2\right)} .
$$

In this case $B$ is induced by the imaginary weak matrix element $\left\langle p_{1 / 2}|W| s_{1 / 2}\right\rangle=i W$. The calculation of the photon circular polarization $P$ arising from the weak interaction is presented in [18].

The circular polarization $P$ appears due to the interference of the amplitudes $A$ and $B$. The relative sign of the $E 1$ and $M 1$ amplitudes depends on the photon circular polarization. One may schematically present this dependence in the total amplitude as $M_{ \pm}=A \pm B$, where "+" and "-" correspond to the right-handed and left-handed circular polarizations. As a result, the average circular polarization is

$$
P \sim \frac{A B^{*}+A^{*} B}{|A|^{2}+|B|^{2}} .
$$

In the case of the weak interaction the factor $i$ in the amplitude $i E 1$ is compensated by the similar factor in the weak matrix element $i W$. In our case the situation is different: if all widths are zero, $A B^{*}+A^{*} B=0$. Thus, the result is proportional to these widths. This difference is explained by the fact that the weak interaction and the circular polarization are time reversal $T$-even while the correlation $\mathbf{E} \cdot \mathbf{B}$ is $T$-odd. Therefore, we need widths which manifest a time asymmetry of the problem.

This model calculation shows us how widths appear in the $\mathbf{E} \cdot \mathbf{B}$ effect for chirality production. It also gives us a very rough estimate for the magnitude of the effect:

$$
\begin{aligned}
P & \sim D \frac{\Gamma \mu e a_{B} \mathbf{B} \cdot \mathbf{E}}{\left(E_{1}-E_{3}\right)^{2}\left(E_{1}-E_{2}\right)} \eta \\
& \sim D^{\prime}[\mathbf{B} / \mathrm{T}] \cdot\left[\mathbf{E} /\left(10^{4} \mathrm{~V} / \mathrm{cm}\right)\right],
\end{aligned}
$$

where $D$ is a numerical coefficient that takes into account (chaotic) suppression of the magnetic and electric dipole matrix elements compared to the Bohr magneton $\mu$ and Bohr radius $a_{B}$ (however, this suppression is more than compensated by a larger enhancement from the very small molecular energy denominators in Eq. (10), see Ref. [14]) and $\eta$ is an asymmetry factor which, as experience with the weak interaction shows, may be quite small. The value for $D^{\prime}$ (overall numerical coefficient depending on $D, \eta$, and energy intervals and widths) strongly depends on the molecule under consideration and can only be reliably determined from experiment or sophisticated molecular structure calculations which are beyond the scope of this work. There is a very dense spectra of Feshbach resonances in collisions of polar molecules; taking modest values for widths and energy intervals from Ref. [11], allowing for suppression of matrix elements, and choosing a value $\eta \sim 10^{-3}$ for the asymmetry factor (see, e.g., Ref. [21]), a rough estimate gives $D^{\prime} \sim 10^{-7}$. The true coefficient for a molecule with dense spectra could be several orders of magnitude larger.

\section{P-EVEN AND P-ODD CORRELATIONS IN COLLISIONS OF COLD MOLECULES}

In principle, $\mathrm{PV}$ and $\mathbf{E} \cdot \mathbf{B}$ circular polarizations in the decay of compound states formed in the collision of cold molecules can be measured. Here we can even have a certain enhancement in comparison with the circular polarization in atoms because of close levels of opposite parity in the compound spectrum - see the small energy denominators in Eqs. (6789). Moreover, in a BEC, Bose-enhancement may lead to almost complete selectivity of the circular polarization of emitted photons due to amplification of the decay of the compound state through the preferred channel (the projection of the angular momentum in the final state of the molecule is different for different circular polarizations of the emitted photon).

There is another manifestation of parity violation in resonance collisions of cold atoms or molecules. It is related to the admixture of an $s$-wave to a $p$-wave compound resonance. For energy $E=0$, only $s$-wave 
molecules have a significant interaction cross section. Consider now a $p$-wave compound resonance. It seems to be invisible for $E=0$. (Note that the $p$-wave amplitude is actually not exactly zero since the trap potential and mean field make the kinetic energy $E$ nonzero even in the ground state.) The weak interaction $W$ mixes states of opposite parity and produces the combined state $|\psi\rangle=|p\rangle+\beta|s\rangle$, thus opening the $s$-wave reaction amplitude proportional to $\beta$. The mixing coefficient $\beta=\langle p|W| s\rangle /\left(E_{s}-E_{p}\right)$ is enhanced since the energy interval between the opposite parity compound states $\left(E_{s}-E_{p}\right)$ is very small due to the high level density in a combined molecule. A similar mechanism is responsible for the enhancement of weak interaction effects in neutron-nucleus reactions $\sim 10^{6}$ times [29] (for a review of the experiments, see $[30]$ ). Interference of the very small $p$-wave amplitude and the weak-induced $s$-wave amplitude leads to PV effects proportional to [29, 31]

$$
P=\sum_{s} \sqrt{\frac{\Gamma_{s}(E)}{\Gamma_{p}(E)}} \frac{i\langle p|W| s\rangle}{\left(E-E_{s}\right)},
$$

where $\Gamma_{p}(E)$ and $\Gamma_{s}(E)$ are capture widths for the $p$ - wave and nearby $s$-wave resonances taken at the actual collision energy $E$ which is assumed to be close to $E_{p_{1 / 2}}$. The kinematic enhancement factor $\sqrt{\Gamma_{s}(E) / \Gamma_{p}(E)}=$ $T_{s} / T_{p} \sim 1 / k R$ tends to infinity at small energies; here $k=p / \hbar \propto \sqrt{E}$ is the molecular wave vector, $R$ is the size of the molecule, $T_{s}$ and $T_{p}$ are $s$-wave and $p$-wave capture amplitudes. PV effects produced in this way involve correlations like $\mathbf{S} \cdot \mathbf{p}$ or $\Gamma\left[\mathbf{n}_{\mathbf{A}} \times \mathbf{n}_{\mathbf{B}}\right] \cdot \mathbf{p}$, where $S$ and $p$ are molecular spin and momentum. There is also a number of parity conserving correlations in the photon emission process, similar to that studied in nuclear reactions with compound resonances [31]. An even richer picture appears in molecules since here we may consider parity violating and parity conserving correlations produced by external fields $\mathbf{E}$ and $\mathbf{B}$.

\section{Acknowledgments}

We are grateful to D. Budker and J. Higbie for useful discussions. This work was supported by the Australian Research Council.
[1] For the first observations of BEC in gases, see: M.H. Anderson et al., Science 269, 198 (1995); C.C. Bradley et al., Phys. Rev. Lett. 75, 1687 (1995); K.B. Davis et al., Phys. Rev. Lett. 75, 3969 (1995).

[2] M. Greiner, C.A. Regal, D.S. Jin, Nature 426, 537 (2003); S. Jochim et al., Science 302, 2101 (2003); M.W. Zwierlen et al., Phys. Rev. Lett. 91, 250401 (2003).

[3] See, e.g., C.A. Regal, C. Ticknor, J.L. Bohn, and D.S. Jin, Nature 424, 47 (2003).

[4] See, e.g., S. Dürr, T. Volz, A. Marte, and G. Rempe, Phys. Rev. Lett. 92, 020406 (2004).

[5] E. Tiesinga, B.J. Verhaar, and H.T.C. Stoof, Phys. Rev. A 47, 4114 (1993).

[6] S. Inouye et al., Nature 392, 151 (1998).

[7] T. Kraemer et al., cond-mat/0512394

[8] V.V. Flambaum and J.S.M. Ginges, cond-mat/0207627

[9] W.C. Stwalley, Phys. Rev. Lett. 37, 1628 (1976); Y.H. Uang, R.F. Ferrante, and W.C. Stwalley, J. Chem. Phys. 74, 6267 (1981).

[10] S.L. Cornish et al., Phys. Rev. Lett. 85, 1795 (2000).

[11] J.L. Bohn, A.V. Avdeenkov, and M.P. Deskevich, Phys. Rev. Lett. 89, 203202 (2002).

[12] A.M. Lane, R.G. Thomas, Rev. Mod.Phys. 30, 257 (1958).

[13] V.V. Flambaum, A.A. Gribakina, G.F. Gribakin, and M.G. Kozlov, Phys. Rev. A 50, 267 (1994); A.A. Gribakina, V.V. Flambaum, and G.F. Gribakin, Phys. Rev E 52, 5667 (1995); V.V. Flambaum, A.A. Gribakina, and G.F. Gribakin, Phys. Rev. A 54, 2066 (1996); V.V. Flambaum, A.A. Gribakina, G.F. Gribakin, Phys. Rev. A 58, 230 (1998); G.F. Gribakin, A.A. Gribakina, and V.V. Flambaum, Aust. J. Phys. 52, 443 (1999).

[14] O.P. Sushkov and V.V. Flambaum, Usp. Fiz. Nauk. 136, 3 (1982) [Sov. Phys. Usp. 25, 1 (1982)]; V.V. Flam- baum and G.F. Gribakin, Prog. Part. Nucl. Phys. 35, 423 (1995).

[15] For general reviews on the possible origins of homochirality, see, e.g., M. Avalos et al., Tetrahedron: Asymmetry 11, 2845 (2000); H. Buschmann, R. Thede, and D. Heller, Angew. Chem. Int. Ed. 39, 4033 (2000).

[16] D.W. Rein, J. Mol. Evol. 4, 15 (1974).

[17] V.S. Letokhov, Phys. Lett. 53A, 275 (1975).

[18] I.B. Khriplovich, Parity nonconservation in atomic phenomena (Gordon and Breach, Philadelphia, 1991).

[19] M.-A. Bouchiat and C. Bouchiat, Rep. Prog. Phys. 60, 1351 (1997).

[20] D.W. Rein, R.A. Hegstrom, and P.G.H. Sandars, Phys. Lett. 71A, 499 (1979); R.A. Hegstrom, D.W. Rein, and P.G.H. Sandars, J. Chem. Phys. 73, 2329 (1980).

[21] J.K. Laerdahl and P. Schwerdtfeger, Phys. Rev. A 60, 4439 (1999).

[22] Ch. Daussy et al., Phys. Rev. Lett. 83, 1554 (1999).

[23] It is not necessary to have all four atoms different. Even such simple molecules as $\mathrm{H}_{2} \mathrm{O}_{2}$ have a chiral structure.

[24] J. Herbig et al., Science 301, 1510 (2003).

[25] E.U. Condon, Rev. Mod. Phys. 9, 432 (1937).

[26] P.L. Polavarapu, Chirality 14, 768 (2002).

[27] M.G. Moore and A. Vardi, Phys. Rev. Lett. 88, 160402 (2002).

[28] Ya.I. Azimov, A.A. Anselm, A.N. Moskalev, and R.M. Ryndin, Sov. Phys. JETP 40, 8 (1975).

[29] O.P. Sushkov and V.V. Flambaum, Pis'ma Zh. Eksp. Teor. Fiz. 32, 377 (1980) [JETP Lett. 32, 352 (1980)].

[30] G.E. Mitchell, J.D. Bowman, and H.A. Widenmuller, Rev. Mod. Phys. 71, 445 (1999).

[31] V.V. Flambaum and O.P. Sushkov, Nucl. Phys. A 435, 352 (1985); Nucl. Phys. A 412, 13 (1984). 